\title{
Produção de cerâmicas celulares por emulsão seguida de gelificação
}

\section{(Production of cellular ceramics by gel casting ceramic emulsions)}

\author{
E. de Sousa ${ }^{1}$, M. Dellú Jr. , V. C. Pandolfelli ${ }^{2}$ F. S. Ortega ${ }^{3}$ \\ ${ }^{I}$ Instituto de Pesquisa e Desenvolvimento - IP\&D, Universidade do Vale do Paraíba -UNIVAP, \\ S. José dos Campos SP 12244-000 \\ ${ }^{2}$ Departamento de Engenharia de Materiais - DEMa, Universidade Federal de S. Carlos - UFSCar, \\ S. Carlos, SP 13565-905 \\ ${ }^{3}$ Departamento de Engenharia Metalúrgica e de Materiais, Centro Universitário da FEI, \\ S. Bernardo do Campo, SP 09850-901 \\ ferortega@fei.edu.br,eliandra.sousa@gmail.com,milton.dellu@embraer.com.br,vicpando@power.ufscar.br
}

\begin{abstract}
Resumo
As cerâmicas celulares têm sido produzidas por diferentes métodos, resultando em uma ampla faixa de estruturas e propriedades. Este trabalho descreve uma nova rota para a produção de cerâmicas celulares, a qual se baseia na gelificação de emulsões constituídas de suspensões cerâmicas aquosas contendo monômeros orgânicos solúveis em água e uma fase líquida apolar emulsificada (querosene). Os efeitos do teor de sólidos e da fração de querosene nas propriedades reológicas das emulsões foram avaliados. A estrutura celular foi analisada por microscopia eletrônica de varredura e revelou poros isolados nas amostras de baixa porosidade, os quais mudaram para uma rede interconectada de poros com o aumento da porosidade. Compressão diametral foi usada para avaliar a resistência mecânica das amostras sinterizadas, a qual variou entre 2,0 e $48 \mathrm{MPa}$, dependendo da porosidade.
\end{abstract}

Palavras-chave: cerâmicas celulares, emulsões o/a, gelcasting.

\begin{abstract}
Cellular ceramics have been produced by several methods, resulting in a wide range of structures and properties. This work describes a novel route to produce cellular ceramics based on the gel casting of emulsions consisting of an aqueous ceramic suspension containing water-soluble organic monomers and an emulsified insoluble liquid phase (kerosene). The effects of solids loading and kerosene content on the rheological properties of emulsions were evaluated. SEM of the cellular structure revealed isolated pores for samples with low porosity, which changed to an interconnected network of pores as the porosity increased. Diametrical compression was used to evaluate the strength of sintered samples, which varied in the range of 2.0 and $48 \mathrm{MPa}$, depending on the porosity.
\end{abstract}

Keywords: cellular ceramics, emulsions o/w, gelcasting, alumina.

\section{INTRODUÇÃO}

Nas últimas décadas o interesse por cerâmicas com estrutura celular, como "honeycombs", espumas e reticulados tem crescido devido a suas propriedades específicas, tais como, baixas densidade e condutividade térmica, estabilidade térmica, elevada área superficial e elevada permeabilidade $[1,2]$. Estas propriedades tornam esta classe de materiais viável para diversas aplicações tecnológicas, como filtros para purificação de gases quentes e de metais líquidos, suportes catalíticos e isolantes térmicos, membranas, sensores de gás, materiais para implantes ósseos, entre outras [3-5]. Vários são os métodos de processamento para a fabricação de cerâmicas celulares $[6,7]$ : réplica de esponjas poliméricas [8,9], produção de espumas a partir de suspensões $[10,11]$, incorporação de material orgânico ao pó cerâmico [12], sinterização controlada para alcançar uma densificação parcial, entre outros. A grande diversidade de processos de fabricação permite obter materiais em ampla faixa de porosidade, além de distribuição de tamanho de células e morfologia variada dos poros. Apesar da grande variedade de métodos de fabricação, o controle preciso da porosidade e da distribuição de tamanho de poros ainda é bastante limitado em alguns dos processos, especialmente naqueles baseados na produção de espuma a partir de uma suspensão [13]. O desenvolvimento de um método capaz de proporcionar estruturas celulares altamente porosas, mais homogêneas, com densidade reprodutível e com reduzido tamanho de poros pode resultar em cerâmicas celulares com propriedades termomecânicas superiores, ampliando suas possibilidades de uso. Neste trabalho uma nova rota de processamento foi usada para a geração das estruturas celulares, o qual consiste na preparação de uma emulsão cerâmica seguida de gelificação [14]. Emulsões são sistemas em que um líquido está disperso 
em outro na forma de gotículas, geralmente estabilizadas por um surfactante (emulsificante), que se localiza na interface entre as fases líquidas, reduzindo a tensão interfacial. As emulsões podem ser classificadas conforme sua fase contínua: água em óleo (w/o) apresenta gotículas de água dispersas na fase óleo contínua, enquanto óleo em água (o/w) consiste em gotículas de óleo dispersas em água [15]. A obtenção de cerâmicas celulares através da gelificação de uma emulsão cerâmica consiste em produzir uma suspensão cerâmica aquosa contendo monômeros orgânicos solúveis em água na qual uma fase líquida apolar é emulsificada. Após a moldagem deste sistema, a gelificação ocorre através da polimerização in situ dos monômeros orgânicos (processo gel casting [5, $10,13]$ ), convertendo-o de um fluido viscoso em um sólido elástico, que é posteriormente seco e submetido ao tratamento térmico. Através desse método pode ser possível controlar com precisão a porosidade do material, que resultam da fração de líquido apolar adicionado à suspensão cerâmica. Acreditase ser possível controlar também o tamanho dos poros através do controle do tamanho das gotículas da fase emulsificada, que dará origem aos poros. A secagem e extração da fase emulsificada apresentam-se como potenciais etapas críticas do processo e requerem que as paredes das células (suspensão cerâmica gelificada) apresentem rigidez elevada para não sofrer grandes deformações durante a remoção da fase apolar, o que poderia causar o colapso dos poros. Adicionalmente, a resistência mecânica deve ser suficiente para suportar as tensões mecânicas decorrentes desta etapa, evitando o surgimento de trincas no material.

O presente trabalho descreve a produção de cerâmicas celulares de alumina por emulsão seguida de gelificação, abordando alguns aspectos importantes do processamento, como o comportamento reológico das emulsões cerâmicas e a caracterização das propriedades físicas, mecânicas e da microestrutura das cerâmicas celulares obtidas. Embora o trabalho tenha sido desenvolvido com, a técnica não se restringe a este material, podendo ser aplicada a outras composições, apenas com alguns ajustes no processamento.

\section{METODOLOGIA EXPERIMENTAL}

Suspensões de alumina (Alcoa, A-1000 SG, $\mathrm{d}_{50}=0,3$ $\mu \mathrm{m})$ com fração volumétrica de sólidos variando entre 0,35 e 0,55 foram preparadas adicionando-se o pó cerâmico a uma solução aquosa contendo $20 \%$ em peso dos seguintes monômeros: N,N,N',N'-hidroximetil acrilamida (HMAM), metacrilamida (MAM), metilenobisacrilamida (MBAM), todos da Sigma-Aldrich, em proporção molar de 3:3:1. Usou-se poliacrilato de amônio como dispersante (Dispersal 130, Lubrizol) em quantidade previamente determinada por curva de defloculação. As suspensões foram desaglomeradas em moinho de bolas por 15 min e usadas para preparar as emulsões. Para isso, as suspensões foram separadas em alíquotas às quais se adicionou querosene (Vimak) sob agitação até a concentração desejada, que variou entre $20 \%$ e $70 \%$ em volume. Nesta etapa, adicionou-se um surfactante (Triton X-114, Sigma) em quantidade suficiente para emulsificar todo o querosene.

O comportamento reológico das emulsões foi caracterizado em reômetro cone-placa (Rheo Stress 300, ThermoHaake) a $25^{\circ} \mathrm{C}$. A taxa de cisalhamento variou entre 0 e $100 \mathrm{~s}^{-1}$, em ciclo de ida e volta. As curvas de tensão $(\tau)$ em função da taxa de cisalhamento $(\dot{\gamma})$ foram ajustadas com bom coeficiente de correlação ao modelo de Herschel-Bulkley (Equação A) [16].

$$
\tau=\tau_{\mathrm{y}}=\mathrm{K}_{\dot{\gamma}^{\mathrm{n}}}
$$

onde $\tau$ é a tensão de cisalhamento $(\mathrm{Pa}), \tau_{\mathrm{y}}$ é a tensão limite de escoamento de Herschel-Bulkley (Pa), K é o índice de consistência (Pa.s), $\dot{\gamma}$ é a taxa de cisalhamento $\left(\mathrm{s}^{-1}\right)$ e $\mathrm{n}$ é a constante de pseudoplasticidade (adimensional), a qual indica o desvio do comportamento plástico ideal ou de Bingham (quando $\mathrm{n}=1$ ).

Para a produção dos corpos-de-prova, quantidades prédeterminadas de catalisador (tetrametiletilenodiamina TEMED, Sigma) e iniciador (persulfato de amônio - APS, Aldrich) foram acrescentadas para promover a gelificação do sistema. Após homogeneização, as emulsões foram vertidas em moldes cilíndricos de PVC com $30 \mathrm{~mm}$ de altura por $18 \mathrm{~mm}$ de diâmetro, onde gelificaram. Os corpos-de-prova foram desmoldados após $24 \mathrm{~h}$ em temperatura ambiente. As amostras foram secas em estufa a $100{ }^{\circ} \mathrm{C}$ e em seguida sinterizadas a $1600{ }^{\circ} \mathrm{C} / 3 \mathrm{~h}$ com taxas de aquecimento de $5{ }^{\circ} \mathrm{C} /$ min até $200{ }^{\circ} \mathrm{C}, 1{ }^{\circ} \mathrm{C} /$ min até $500{ }^{\circ} \mathrm{C}$ e $5{ }^{\circ} \mathrm{C} /$ min até a temperatura de sinterização. $\mathrm{O}$ resfriamento seguiu-se inercialmente até a temperatura ambiente.

A densidade aparente e a porosidade aparente das amostras sinterizadas foram determinadas pelo princípio de Arquimedes em água [17]. A distribuição de tamanho de poros foi avaliada por porosimetria de mercúrio (Micromeritics - Poresizer 9320). A microestrutura e morfologia das cerâmicas celulares foram analisadas por microscopia eletrônica de varredura (MEV, Philips, XL-30).

A resistência mecânica foi avaliada em uma máquina de ensaios universal (Mohr \& Federhaff AG), na temperatura ambiente, por ensaio de compressão diametral. Foram utilizados corpos cilíndricos com $18 \mathrm{~mm}$ de diâmetro, obedecendo a uma relação geométrica entre altura e diâmetro de $0,3<\mathrm{h} / \mathrm{d}<1,5$, onde $\mathrm{h}$ é a altura e d é o diâmetro do cilindro, conforme a norma ASTM C496-90 [18]. A velocidade de carregamento aplicada foi de aproximadamente $0,5 \mathrm{~mm} / \mathrm{min}$. Os dados experimentais de resistência mecânica à compressão foram ajustados pela equação B, proposta por Gibson-Ashby (GA) [19].

$$
\frac{\sigma_{o}}{\sigma_{s}}=C_{1}\left(\frac{\rho_{0}}{\rho_{s}}\right)^{n}
$$

onde $\sigma_{\mathrm{o}}$ é a resistência do material celular, $\sigma_{\mathrm{s}}$ é resistência da fração sólida, $\rho_{\mathrm{o}} / \rho_{\mathrm{S}}$ é a densidade relativa $\left(\rho_{\mathrm{o}}\right.$ é a densidade do material celular e $\rho_{\mathrm{s}}$ é a densidade da fração sólida, adotada como $3,95 \mathrm{~g} / \mathrm{cm}^{3}$ neste trabalho), $\mathrm{C}_{1}$ é uma constante relacionada com a forma da célula e o expoente $n$ 
é uma constante igual a 1,5 para uma estrutura constituída por células cúbicas.

\section{RESULTADOS E DISCUSSÃO}

Caracterização reológica das emulsões cerâmicas

As curvas de tensão contra taxa de cisalhamento das suspensões e emulsões cerâmicas contendo diferentes frações volumétricas de sólidos $(0,35-0,55)$ e adições de querosene (20-70\%-vol.) são mostradas na Fig. 1. Todas as emulsões apresentaram comportamento pseudoplástico e algumas delas, especialmente aquelas com alta concentração de querosene $(0,35-70 q, 0,50-50 q, 0,50-70 q, 0,55-40 q$ e $0,55-$ $50 \mathrm{q} ; \mathrm{q}=\%$ vol. de querosene), apresentaram comportamento dependente do tempo (tixotrópico), caracterizado por uma
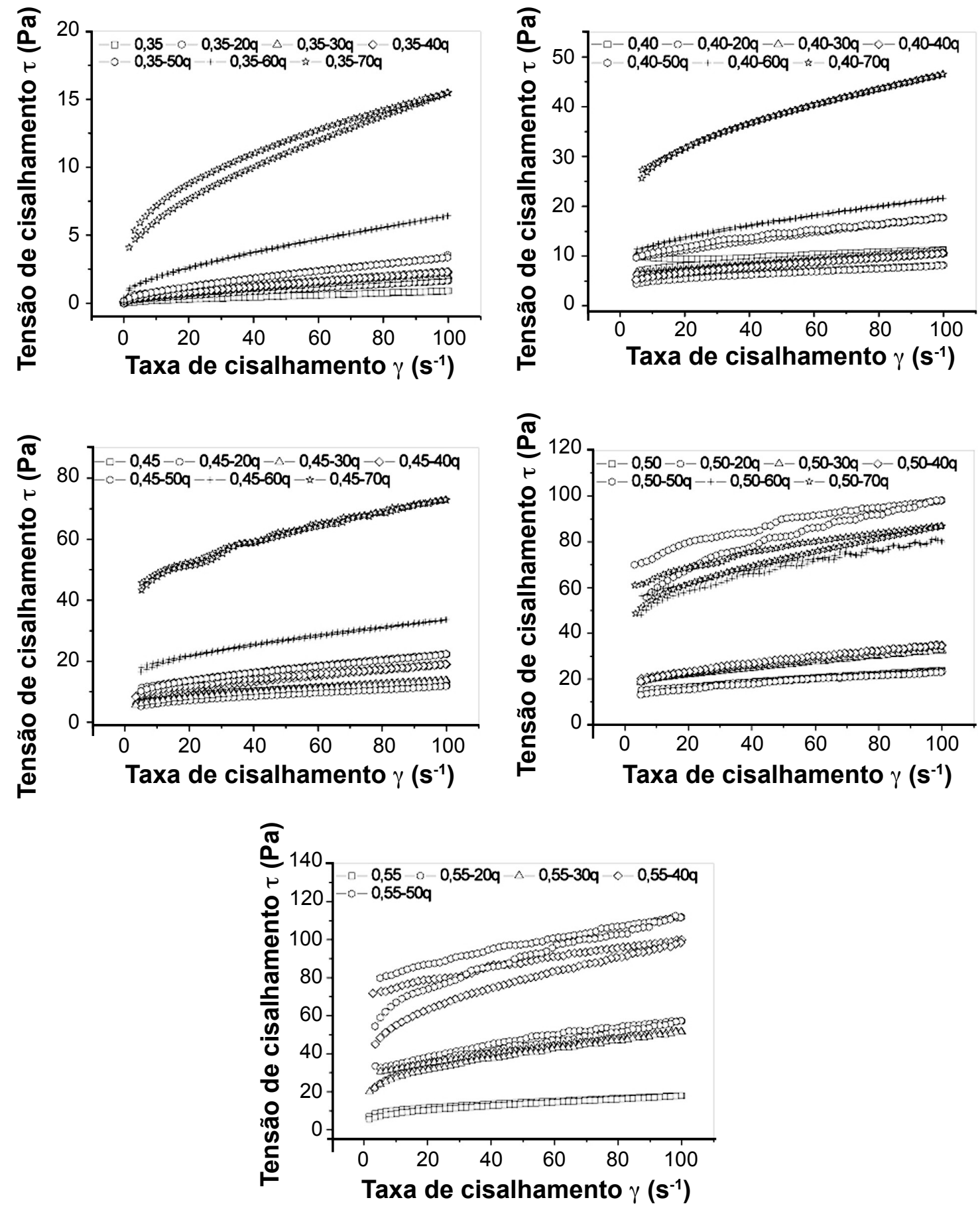

Figura 1: Curvas de fluxo para suspensões cerâmicas e emulsões contendo diferentes frações volumétricas de sólidos na suspensão (0,350,55 vol.) e frações volumétricas de querosene emulsificada (20q-70q \%vol.).

[Figure 1: Flow curves for ceramic suspensions and emulsions containing different solids loading in the suspension (0.35-0.55 vol.) and volumetric fraction of emulsified kerosene (20q-70q vol.\%).] 
histerese na curva de carregamento-descarregamento. Para a suspensão com $0,55 \mathrm{vol}$. de sólidos foi possível efetuar medidas com adições de até $50 \%$ vol. de querosene. Acima deste valor, as emulsões tornaram-se excessivamente viscosas.

A partir das curvas mostradas na Fig. 1, foram calculados os parâmetros $\tau_{\mathrm{y}}$, K e n, conforme o modelo de HerschelBulkley (Equação $\mathrm{A}$ ). Os valores de $\mathrm{n}$ variaram entre 0,439 para emulsões com alta concentração de querosene e 0,820 para suspensões sem querosene, confirmando o comportamento pseudoplástico, no qual $\mathrm{n}<1$. A Fig. 2 mostra a variação da tensão de escoamento $\left(\tau_{\mathrm{y}}\right)$ em função da fração volumétrica de querosene na emulsão, para suspensões com diferentes concentrações de sólidos.

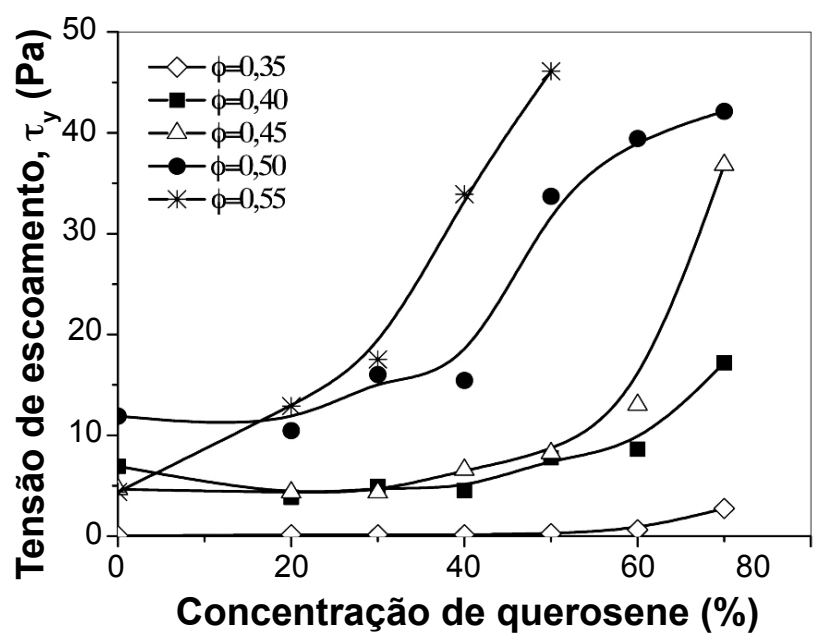

Figura 2: Tensão de escoamento $\left(\tau_{\mathrm{y}}\right)$ das emulsões cerâmicas com diferentes concentrações de sólidos na suspensão $(\phi)$ em função da fração volumétrica de querosene emulsificada.

[Figure 2: Yield stress $\left(\tau_{\mathrm{y}}\right)$ of ceramic emulsions with different solids loadings in the suspension ( $\phi)$ as a function of volume fraction of emulsified kerosene.]

Observa-se que a tensão de escoamento aumenta com a fração de querosene adicionada, sendo este aumento mais acentuado para as emulsões produzidas a partir de suspensões com alta concentração de sólidos. Ao contrário de suspensões de partículas sólidas, em que a tensão de escoamento tende a desaparecer em sistemas bem dispersos [20], a tensão de escoamento é uma característica observada frequentemente em emulsões [21, 22]. Significa que para tensões de cisalhamento abaixo da tensão de escoamento, ocorre a deformação elástica e reversível das gotículas. A capacidade de resistir ao escoamento resulta da tensão interfacial existente entre as gotículas e a fase contínua. A variação da energia livre de Gibbs associada à interface líquido-líquido é dada por [23]:

$$
\Delta \mathrm{G}=\gamma_{\text {liq-liq }} \cdot \Delta \mathrm{A}
$$

onde $\gamma_{\text {liq-liq }}$ é a tensão interfacial, $\Delta \mathrm{G}$ é a variação da energia livre de Gibbs associada às interfaces e $\Delta \mathrm{A}$ é a variação da área interfacial.
A deformação das gotículas causa um aumento da interface líquido-líquido elevando a energia livre de Gibbs, originando uma tendência de retorno das gotículas à forma de menor área interfacial, que se traduz macroscopicamente no comportamento elástico observado abaixo da tensão de escoamento. Em geral, quanto maior a área interfacial, maior será a tendência à recuperação elástica. Adicionalmente, as moléculas do surfactante usado para estabilizar a emulsão formam uma película elástica, cujo comportamento decorre da atração de van de Waals entre as frações hidrofóbicas das moléculas [15, 21, 22], o que também contribui para o comportamento observado. Isso explica o aumento da tensão de escoamento com a fração de fase emulsificada. Deve-se considerar ainda que a grande maioria dos trabalhos que abordam a reologia de emulsões considera sistemas em que a fase contínua é formada por um líquido puro, ao passo que neste trabalho a fase contínua constitui-se de uma dispersão de partículas sólidas em meio líquido, o que torna mais complexa a análise do comportamento reológico.

A viscosidade aparente $\left(\eta_{\text {ap }}\right)$ das emulsões cerâmicas, dada pela razão entre a tensão $(\tau)$ e a taxa de cisalhamento $(\dot{\gamma})$, é mostrada na Fig. 3 em função do teor de querosene, para $\dot{\gamma}$ igual a $50 \mathrm{~s}^{-1}$. Nota-se que $\eta_{\text {ap }}$ aumenta com a concentração de sólidos e com a fração volumétrica de querosene. Para as emulsões com fração volumétrica de sólidos entre 0,35 e 0,45 o aumento na viscosidade é mais acentuado para concentrações de querosene acima de $60 \%$ vol. Já para as emulsões com fração volumétrica de sólidos de 0,50 e 0,55 o aumento na viscosidade é notório mesmo para frações mais baixas de querosene (40 e 50\%-vol.).

O comportamento da viscosidade de suspensões em

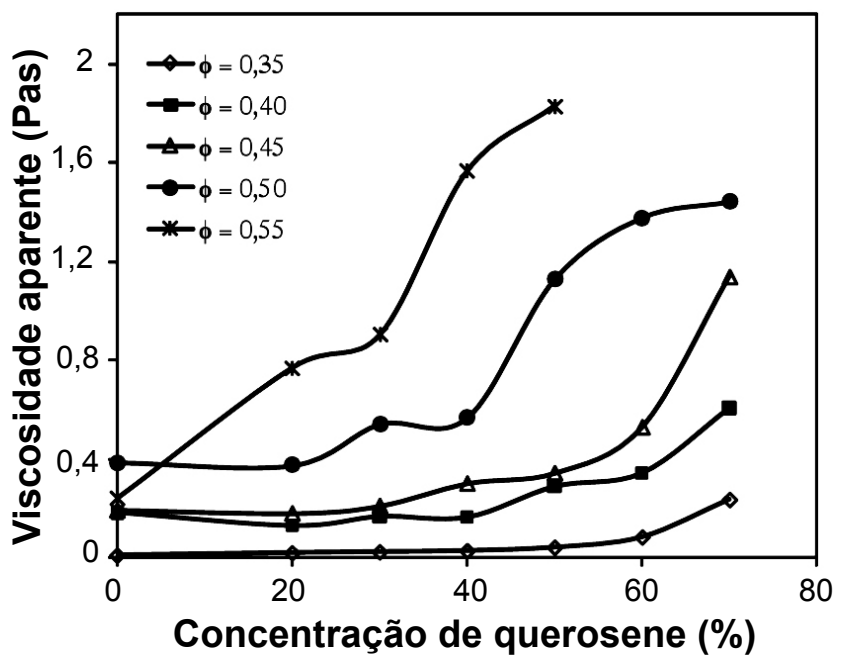

Figura 3: Viscosidade aparente de emulsões com diferentes concentrações de sólidos na suspensão $(\phi)$ em função da fração volumétrica de querosene emulsificada, medida a $\dot{\gamma}=50 \mathrm{~s}^{-1}$.

[Figure 3: Apparent viscosity of emulsions with different solids loadings in the suspension ( $\phi)$ as a function of volume fraction of emulsified kerosene, measured at $\dot{\gamma}=50 \mathrm{~s}^{-1}$.]

função da concentração de partículas sólidas tem sido bem estudado, sendo descrito por alguns modelos semi-empíricos, como o de Krieger e Dougherty e o de Quemada [20]. Nestes 
modelos, a viscosidade relativa de suspensões aumenta lentamente com a fração volumétrica de sólidos mas, à medida que esta se aproxima da fração máxima de sólidos, passa a crescer rapidamente, tendendo assintoticamente para o infinito. Estudos demonstram que a viscosidade de emulsões varia em função da concentração da fase dispersa de modo semelhante: a viscosidade relativa aumenta com a fração volumétrica $(\phi)$ da fase emulsificada, dependendo de fatores como a distribuição de tamanho das gotículas, tensão interfacial e razão entre a viscosidade da fase contínua e da fase dispersa, entre outros. À semelhança de suspensões de partículas sólidas, este valor aumenta acentuadamente à medida que $\phi$ se aproxima da fração máxima $\left(\phi_{\mathrm{m}}\right)$, o qual depende da distribuição de tamanho das gotículas. Por esta razão, alguns autores tentaram aplicar os modelos citados para descrever a viscosidade de emulsões em função da fração volumétrica emulsificada. No entanto, ao contrário de suspensões de partículas sólidas, as emulsões são formadas por gotículas deformáveis, tendo sido melhor descritas pela equação (D) [24].

$$
\eta_{R}=\left(1-\frac{A_{0}+A_{1} \log R e}{\varphi_{m}^{0.5}}\right)^{-[\eta]}
$$

onde $A_{0}$ e $A_{1}$ são constantes, $\varphi_{m}$ é a máxima fração de fase emulsificada, [ๆ] é a viscosidade intrínseca do sistema e $\mathrm{Re}$ é o número de Reynolds, dado por, $R e=\rho_{c} \dot{\gamma} R^{2} / \eta_{c}$ onde $\rho_{c}$ e $\eta_{c}$ são a densidade e a viscosidade da fase contínua, respectivamente, $\mathrm{R}$ é o raio das gotículas e $\dot{\gamma}$ é a taxa de cisalhamento aplicada. As constantes $\mathrm{A}_{0}$ e $\mathrm{A}_{1}$ aumentam linearmente $\operatorname{com} \varphi$ [25], de modo que as equações (D) apresentam alguma similaridade com os modelos adotados para suspensões. No entanto, a equação (D) leva em consideração o efeito da taxa de cisalhamento aplicada, a qual deforma as gotículas da fase dispersa, alterando o valor de $\eta_{R}$. A deformabilidade das gotículas emulsificadas favorece o comportamento pseudoplástico das emulsões, mesmo com baixas concentrações de fase emulsificada, como foi observado na Fig. 1.

Assim, o comportamento reológico de emulsões produzidas a partir de suspensões deverá ser estudado, em trabalhos futuros, com base em modelos mais sofisticados, que considerem a influência tanto da concentração de sólidos na suspensão como da fração volumétrica da fase emulsificada.

Deve-se ressaltar que elevados valores de tensão de escoamento resultam em maior viscosidade aparente, como mostram as Figs. 2 e 3. Do ponto de vista do processamento do material, esta característica favorece o aprisionamento de bolhas de ar durante a moldagem das emulsões, que se tornam defeitos concentradores de tensão, prejudicando as propriedades mecânicas do material. O uso de procedimentos que evitem o aprisionamento de bolhas durante a mistura e moldagem da emulsão deve resultar em significativa melhora nas propriedades mecânicas do material.

\section{Caracterização das cerâmicas celulares}

A porosidade aparente das amostras sinterizadas em função da fração de querosene é mostrada na Fig. 4. A faixa de porosidade variou entre 10-64\%. Como esperado, a porosidade aparente aumenta com a fração de querosene. Destaca-se a boa correlação entre a fração volumétrica de querosene e a porosidade do material, o que possibilita um controle preciso da porosidade do material. Observase também que para uma mesma fração de querosene a porosidade das amostras é afetada pela concentração de sólidos da suspensão. Suspensões com elevados teores de sólidos resultam em cerâmicas celulares com valores mais elevados de porosidade. De fato, à medida que a concentração de sólidos na suspensão aumenta, a relação entre o volume de querosene e o volume de sólidos torna-se mais elevada, resultando em uma porosidade superior após a remoção do querosene.

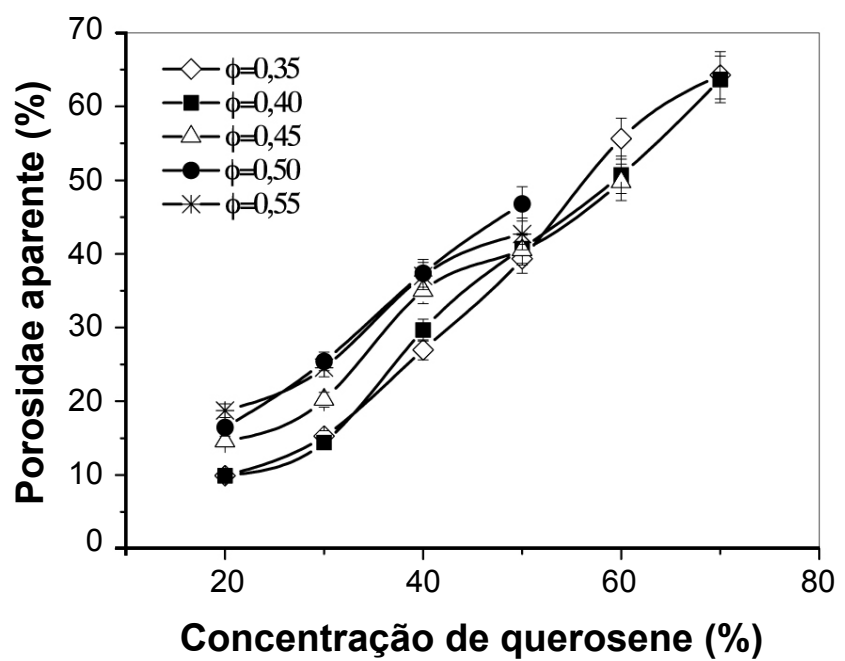

Figura 4: Porosidade aparente das cerâmicas celulares sinterizadas produzidas por emulsão em função da fração de querosene.

[Figure 4: Apparent porosity of sintered cellular ceramics produced by emulsion as a function of the kerosene fraction.]

O volume de mercúrio intrudido por unidade de massa em função do diâmetro do poro é mostrado na Fig. 5 para as amostras produzidas com a suspensão contendo fração volumétrica de sólidos de 0,50 e concentração volumétrica de querosene entre $20 \%$ e $50 \%$. O gráfico mostra a ocorrência de poros com distribuição de tamanho estreita e diâmetro abaixo de $2 \mu \mathrm{m}$, o qual diminui nas amostras menos porosas.

Deve-se ressaltar que a porosimetria de mercúrioé baseada em um modelo que admite amostras com poros cilíndricos, cujo diâmetro é calculado a partir da pressão necessária para causar a penetração do mercúrio no poro. Este modelo não prevê a existência de poros relativamente grandes com aberturas pequenas, denominados genericamente de poros em forma de garrafa. Quando a amostra apresenta poros com esta característica, o modelo calcula, na verdade, o diâmetro do canal que liga poro à superfície, e não seu diâmetro real. De fato, a Fig. 6 mostra a microestrutura de amostras com 


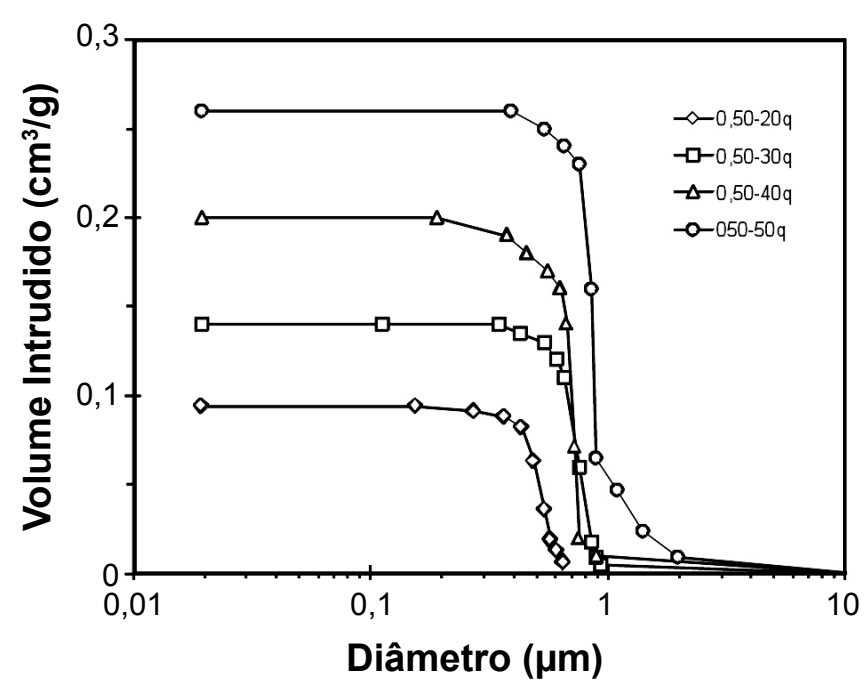

Figura 5: Distribuição de tamanho de poro das cerâmicas celulares produzidas por emulsão $(\phi=0,50)$ com diferentes frações volumétricas de querosene, medido por porosimetria de mercúrio. [Figure 5: Pore size distribution of cellular ceramics produced by ceramic emulsion $(\phi=0.50)$ with different volume fractions of kerosene, measured by mercury porosimetry.]

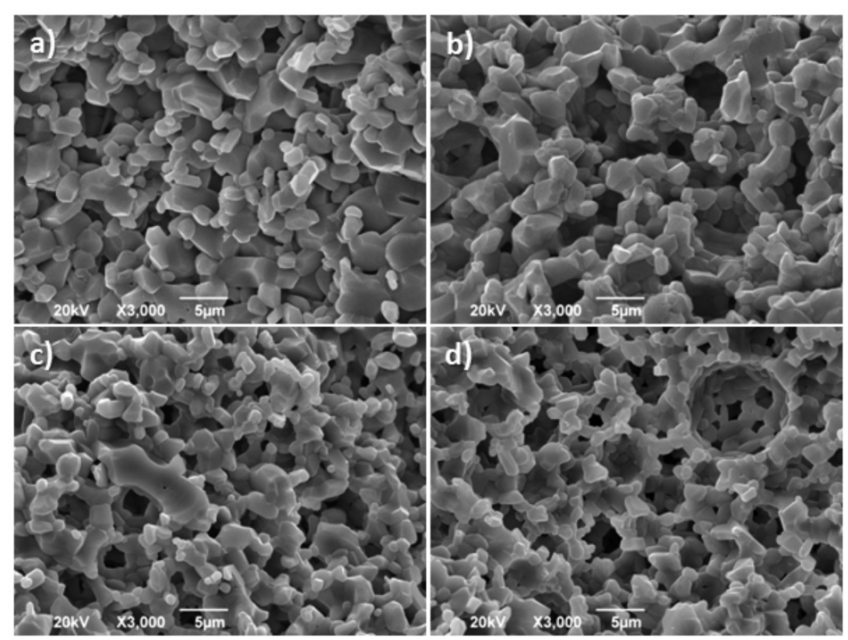

Figura 6: Micrografias, por microscopia eletrônica de varredura, das cerâmicas celulares produzidas por emulsão $(\phi=0,50) \mathrm{em}$ função da fração de querosene: a) $20 \%$-vol.; b) $30 \%$-vol.; c) $40 \%$ vol.; d) $50 \%$-vol.

[Figure 6: SEM micrographs of cellular ceramics produced by ceramic emulsion $(\phi=0.50)$ as a function of the kerosene fraction: a) 20\%-vol.; b) 30\%-vol.; c) 40\%-vol.; d) 50\%-vol.]

diferentes porosidades obtidas a partir da suspensão com fração volumétrica de sólidos de 0,50 .

Em todas as amostras observa-se uma distribuição homogênea de poros, com diâmetro variando entre 2 e 10 $\mu \mathrm{m}$, aproximadamente. Nota-se também que, à medida que a porosidade aumenta, os poros tendem a se tornar esféricos e interconectados por aberturas nas paredes das células. Esta característica explica o fato de que o diâmetro dos poros observados na Fig. 6 é em geral maior do que o diâmetro medido por porosimetria de mercúrio (Fig. 5).

Comparando-se com cerâmicas celulares produzidas por réplica ou por produção de espuma, os valores de porosidade atingidos foram relativamente baixos. No entanto, a extensão do estudo, com novas combinações de surfactante e de líquido emulsificado pode resultar em valores mais elevados de porosidade, uma vez que a preparação de emulsões com mais de $90 \%$ de fase emulsificada é amplamente descrita na literatura [15, 21, 22, 25].

A Fig. 7 apresenta os valores médios de resistência à compressão das cerâmicas celulares produzidas com as suspensões contendo diferentes concentrações de sólidos em função da densidade relativa $\left(\rho / \rho_{0}\right)$, onde $\rho$ é a densidade do material celular e $\rho_{0}$ é a densidade das paredes que formam as células, estimada em $3,95 \mathrm{~g} / \mathrm{cm}^{3}$.

Em todos os casos, a resistência à compressão aumenta com a densidade relativa. Através de uma regressão sobre os pontos mostrados na Fig. 7, obtiveram-se os valores referentes ao produto $\sigma_{\mathrm{s}} \times \mathbf{C}_{1}$ e $\mathbf{n}$, conforme o modelo de Gibson-Ashby (Equação B), os quais foram 75,5 e 2,6 respectivamente. Embora o valor de $\mathrm{n}$ tenha sido superior àquele proposto pelo modelo Gibson-Ashby, foi compatível com resultados encontrados para diversas cerâmicas celulares, tais como alumina-mulita $(n=2,2)$ [26], cordierita $(n \cong 3)$ [27] e oxicarbeto de sílicio $(n=3,6)$ [28]. De fato, a Equação B é baseada em um arranjo de células cúbicas formadas por 12 elementos retilíneos cuja fratura ocorre devido ao momento fletor transferido por células vizinhas. Entretanto, a estrutura observada em diversas cerâmicas celulares difere em maior ou menor grau da estrutura considerada na dedução do modelo, de modo que tensões axiais não consideradas no modelo original podem somarse ao momento fletor, resultando em valores do expoente $n$ diferentes de 1,5 [29].

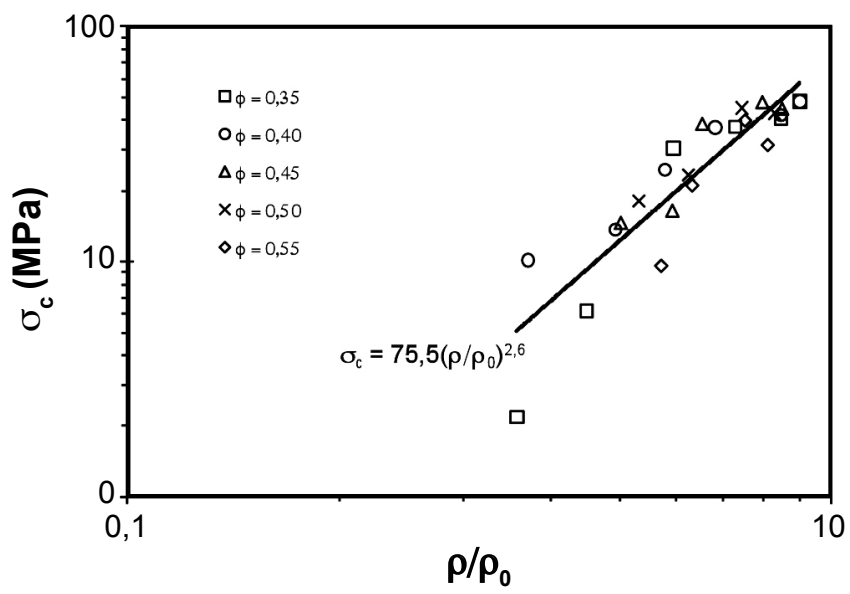

Figura 7: Tensão de ruptura das cerâmicas celulares produzidas por emulsão em função da fração de querosene.

[Figure 7: Fracture strength of cellular ceramics produced by ceramic emulsion as a function of the kerosene fraction.]

\section{CONCLUSÕES}

Cerâmicas celulares à base de alumina foram obtidas por meio de uma nova rota de processamento, a emulsificação 
de uma fase apolar em uma suspensão aquosa de partículas cerâmicas, seguida de gelificação, secagem e sinterização.

A concentração de sólidos na suspensão e a quantidade de querosene emulsificada exerceram forte influência sobre o comportamento reológico das emulsões cerâmicas, elevando os valores de viscosidade aparente e de tensão de escoamento, com conseqüências importantes na etapa de moldagem. As cerâmicas celulares apresentaram excelente correlação entre porosidade e concentração de querosene emulsificada, mostrando um importante aspecto da rota proposta, o controle preciso da porosidade. O processo possibilitou a fabricação de cerâmicas celulares com porosidade na faixa de $10-64 \%$. Suspensões com maior concentração de sólidos resultam em maior porosidade, para uma dada fração de querosene emulsificado, devido à maior relação querosene/material cerâmico. A resistência mecânica variou com a porosidade entre 2 e $48 \mathrm{MPa}$. Amostras produzidas no limite superior de concentração de sólidos e de fração emulsificada apresentaram resistência mecânica reduzida pela maior incidência de defeitos de moldagem, decorrentes da elevada tensão de escoamento. $\mathrm{O}$ eventual aprimoramento da técnica de moldagem pode resultar em ganho significativo de resistência mecânica. Os poros apresentaram geometria tendendo à esfericidade e diâmetro abaixo de $10 \mu \mathrm{m}$, sendo interconectados por aberturas nas paredes das células. Estudos adicionais devem ser conduzidos com o intuito de elevar a fração emulsificada através do estudo da interface suspensão-querosene, de reduzir defeitos de moldagem, especialmente em sistemas com alta tensão de escoamento e de investigar o efeito da taxa de cisalhamento durante a emulsificação sobre a distribuição de tamanho de poros do material. Embora o estudo tenha sido desenvolvido com alumina, não há qualquer particularidade do processo que o restrinja a este material, podendo ser estendido a outras composições cerâmicas com alguns ajustes, como na dispersão do pó e na sinterização. O conjunto de propriedades observado sugere que o material poderá apresentar bom desempenho em aplicações termomecânicas, o que deverá ser melhor investigado em estudos futuros.

\section{AGRADECIMENTOS}

Os autores agradecem ao apoio recebido do $\mathrm{CNPq}$.

\section{REFERÊNCIAS}

[1] M. Scheffler, P. Colombo (Eds.), "Cellular Ceramics: Structure, Manufacturing, Properties and Applications", Wiley VCH, Germany (2005).

[2] P. Colombo, E. Bernardo, Composites Sci. Tech. 63 (2003) 2353.

[3] P. Ciambelli, V. Palma, P. Russo, S. Vaccaro, Catalysis Today 75 (2002) 471.

[4] M. D. M. Innocentini, P. Sepulveda, V. R. Salvini, V. C.
Pandolfelli, J. Am. Ceram. Soc. 81, 12 (1998) 3349.

[5] P. Sepulveda, Am. Ceram. Soc. Bull. 76 (1997) 61.

[6] L. Montanaro, Y. Jorand, G. Fantozzi, A. Negro, J. Eur. Ceram. Soc. 18 (1998), 1339.

[7] A. R. Studart, U. T. Gonzenbach, E. Tervoort, L. J. Gauckler, J. Am. Ceram. Soc. 89, 6 (2006) 1771.

[8] K. Schwartzwalder, A. V. Somers, U.S. Patent 3,090,094 (1963).

[9] J. Saggio-Woyansky, C. E. Scott, W. P. Minnear, Am. Ceram. Soc. Bull. 71, 11 (1992) 1674.

[10] P. Sepulveda, J. G. P. Binner, J. Eur. Ceram. Soc. 19 (1999) 2059.

[11] H. X. Peng, Z. Fan, J. R. G. Evans, J. J. C. Busfield, J. Eur. Ceram. Soc. 20 (2000) 807.

[12] T. J. Fiztgerald, A. Mortensen, V. J. Michaud, J. Mater. Sci. 30 (1995) 1037.

[13] F. S. Ortega, P. Sepulveda, M. D. M. Innocentini, V. C. Pandolfelli, Am. Ceram. Soc. Bull. 80 (2001) 37.

[14] E. Sousa, M. Dellú Júnior, V. C. Pandolfelli, F. S. Ortega, Mater. Sci. Forum 591-593 (2008) 498.

[15] F. Leal-Calderon, V. Schmitt, J. Bibette, "Emulsion Science: Basic Principles", $2^{\text {nd }}$ Ed., Springer, New York, EUA (2007).

[16] C. W. Macosko, "Rheology: Principles, Measurements and Applications", VHC, New York, EUA (1994).

[17] Am. Soc. for Testing and Materials, ASTM C 496-90: "Standard test method for water absorption, bulk density, apparent porosity and apparent specific gravity of fired whiteware products", Philadelphia, EUA (1988).

[18] Am. Soc. for Testing and Materials, ASTM C 37388: "Standard test method for splitting tensile strength of cylindrical concrete specimens", Philadelphia, EUA (1990). [19] L. J. Gibson, M. F. Ashby, "Cellular solids: structure and properties", 2 ${ }^{\text {nd }}$ Ed., Cambridge University Press, Cambridge, UK (1997).

[20] Z. Zhou, P. J. Scales, D. V. Boger, Chem. Eng. Sci. 56 (2001) 2901.

[21] R. Pal, Food Hydrocoll. 20 (2006) 997.

[22] N. Jager-Lézer, J. F. Tranchant, V. Alard, C. Vu, P. C. Tchoreloff, J. L. Grossiord, Rheol. Acta 37 (1998) 129.

[23] D. H. Everett, "Basic Principles of Colloid Science", The Royal Society of Chemistry, Cambridge, UK (1988).

[24] R. Pal, Coll. Surf.: Physicochem. Eng. Aspects 137 (1998) 275.

[25] K. K. Krynke, J. P. Sek, Coll. Surf. A: Physicochem. Eng. Aspects 245 (2004) 81.

[26] R. Brezny, D. J. Green, J. Am. Ceram. Soc. 76, 9 (1993) 2185.

[27] F. A. C. Oliveira, S. Dias, M. F. Vaz, J. C. Fernandes, J. Eur. Ceram. Soc. 26 (2006) 179.

[28] P. Colombo, J. R. Hellmann, D. L. Shelleman, J. Am. Ceram. Soc. 84, 10 (2001) 2245.

[29] F. S. Ortega, J. A. Rodrigues, V. C. Pandolfelli, Am. Ceram. Soc. Bull. 83 (2004) 9501.

(Rec. 14/01/2010, Rev. 19/03/2010, Ac.07/05/2010) 